\title{
GEOREFERENCED FOUR-DIMENSIONAL VIRTUAL ENVIRONMENTS: PRINCIPLES AND APPLICATIONS
}

\author{
J. Raper ${ }^{1}$, T. McCarthy and N. Williams \\ Department of Geography, Birkbeck College, University of London, \\ 7-15 Gresse Street, London W1P 2LL, U.K.
}

\begin{abstract}
This paper introduces and defines the concept of a four-dimensional virtual environment as "multidimensional/multimedia representations of phenomena in natural and built environments permitting the realistic monitoring, analysis and evaluation of the component phenomena". It is argued that current geographic information systems cannot handle these representations and so new tools need to be built. This paper describes two applications of virtual environments: the first is an application which can link oblique or vertical aerial videography to map or surface models in real-time, while the second example is an application which links a user-browseable virtual world with a map. The paper concludes by suggesting that the approaches demonstrated here are indicative of what the next generation of geographic information handling might look like. (C) 1999 Elsevier Science Ltd. All rights reserved
\end{abstract}

\section{INTRODUCTION}

Organisations managing land, facilities and resources have an ongoing requirement for timely sources of geographical information, effective visualisation and powerful analytical systems in order to more effectively manage their operations. To date, much geographical data acquisition and handling has been two-dimensional in nature, perhaps due to the fact that most data sources are derived from paper maps. Until quite recently (Raper \& McCarthy, 1997), little attempt has been made to manage geographic information using true three-dimensional (3D) or four-dimensional (4D) representations such as surface models, real-time data, process models or virtual worlds and to develop new forms of analysis for them. Multimedia or multidimensional data types in current geographic information systems (GIS) are limited to images or video clips linked to a single piece of geometry or to static terrain surface display.

This paper suggests that the creation of georeferenced 4D virtual environments using such new representations is a natural progression from the approach offered by contemporary GIS (Raper, 1995). This new approach is characterised chiefly by an

\footnotetext{
${ }^{1}$ Requests for reprints should be addressed to Jonathan Raper, Department of Geography, Birkbeck College, University of London, 7-15 Gresse Street, London W1P 2LL, U.K.
} 
ability to handle georeferenced multidimensional and multimedia data types together in an integrated virtual environment. Virtual environments can be defined as "multidimensional/multimedia representations of phenomena in natural and built environments permitting the realistic monitoring, analysis and evaluation of the component phenomena". A whole new generation of geographic information management problems can be addressed if the dimensions and modality of representation are extended. The enrichment of spatial representations could be viewed as one of the priority projects for the emerging discipline of geographic information science.

Examples of the data types which can be used to create virtual environments include georeferenced videography, real-time positioning, surface and subsurface 3D modelling and dynamic process modelling. Taken together, these data types can be used to create dynamic virtual environments. Given the diversity of these data types it seems unlikely that any single new system will prove able to accommodate them all. Accordingly, it is suggested in this paper that new functionality for the manipulation of these data types should be developed as separate component applications with standard interfaces which can interoperate using georeferenced data in a distributed network computing environment.

Two example applications to illustrate how these new data types can be used to construct 4D virtual environments are presented in this paper. The first is an application which can link oblique or vertical aerial videography to map and surface models in realtime. The virtual environment created here is one in which places/features viewed in the oblique or vertical video can be positioned absolutely and added to a spatial database of target features with the source image so that times and places are linked and indexed. Potential applications include environmental monitoring, for example, after storm/flood events when rapid identification of management problems is needed, or utility asset monitoring where potentially dangerous faults have to be located and corrected quickly in nonurban settings. The development of such videography applications depends on the development of airborne data-acquisition systems including georeferenced digital-video, real-time positioning, attitude and ranging systems and field dataloggers. Aerial videometry is used to enable features viewed in aerial video to be incorporated into georeferenced virtual environments.

The second example is an application which links a user-browsable virtual world with a map. In this case the virtual environment is a surface model draped with a variety of userselectable forms of information which can be viewed from any angle or position, and in which the viewpoint/field of view in the virtual world is shown on the linked map. Potential applications for this form of virtual environment include real-time monitoring of the position of a moving vehicle where the user can examine the phenomena of interest using two different visualisation tools at the same time. The development of this application requires the development of spatially linked views of the target in a GIS and a virtual reality (VR) system. The resultant system could be installed locally on users' machine or alternatively accessed from the World Wide Web.

\section{IMPLEMENTATION DESIGN FOR THE APPLICATIONS}

To develop applications of 4D virtual environments requires a space- and timereferenced framework within which to re-create the behaviours of the phenomena under 
study. However, at present no such framework exists: current GIS have a limited ability to handle multimedia and multidimensional data types. Two potential approaches can be identified. Firstly, there is the development of a new global data model for the handling of georeferenced multimedia and multidimensional data using object-oriented techniques. This is practical and necessary but a long-term objective. Secondly, there is the development of application-specific architectures that develop new spatially and temporally extended referencing frameworks - which is the approach taken in this paper. Such application-specific architectures are easiest to implement as components which interoperate which each other.

When developing such "extended-referencing" architectures based upon component interoperability, each component needs to be able to work with a space and time framework understood by all the components. In the case of space, this can be any local or global coordinate system which is easy to implement; in the case of time, it can be any common unit of time - such as the second which all the components can handle (note that the Global Positioning System [GPS] uses this unit). Given the strict adoption of these spatial and temporal units, each component can exchange information on a common basis. However, the key implementational challenge for $4 \mathrm{D}$ virtual environments is that of temporal latency, that is, handling the time delays between data capture from different sources.

The following sections describe the components developed to support the two virtual environment applications discussed in this paper. As the data to develop virtual environments frequently does not exist, the process of gathering data and constructing the components is also covered in the following sections.

\section{COMPONENT TECHNOLOGIES FOR AN AERIAL VIDEOGRAPHY VIRTUAL ENVIRONMENT}

To create a virtual environment combining aerial video imaging with mapping requires the positioning of the moving platform in space and time. Maps have geographic coordinates built in making it desirable to position the platform and any phenomena visible in the video imagery using map coordinates. As video has time units built in it is desirable to assign temporal attributes to the positions recorded for the platform and any phenomena visible. This can be done by recording geographic positions with the video signal and by recording times for positions at data capture time. The data capture system used in this application example is made up of four main modules: georeferenced video; positioning, attitude and ranging systems; datalogging systems; and videometry (measuring position of features visible in video imagery).

\section{Georeferenced Videography}

Normal colour video imaging has become a recognised and valuable new technique of remote sensing in the 1990s (King, 1995; Mausel, Everitt, Escobar, \& King, 1992). Most video imagery is still predominantly analogue in form (at the time of writing), being recorded by the camera as brightness/colour levels line by line at 25 frames per second and modulated onto a waveform which is then transmitted to a remote receiver or recorded onto tape. In this paper, georeferenced videography is understood to mean the linkage of 
positional information such as GPS to individual video frames (Cooper, McCarthy, \& Raper, 1995). The GPS positional information can be stored with a frame once per second either using the audio track (Paragee, 1997) or by using the interframe vertical line interval (VLI; Raper \& McCarthy, 1994a). There are various advantages for each of these methods including efficient data management and, in the case of the latter, the ability to securely transmit data from camera platform to a control centre in real-time using microwave signals if necessary.

The aerial videography captured for this application was obtained obliquely using a Hi8 Pulnix camera and had the data for the platform position encoded into the VLI of the video frame using a Navtech Telenav ${ }^{\mathrm{TM}}$ hardware encoder (Navtech, 1997). After the flight, the recorded positional data was decoded by a Telenav ${ }^{\mathrm{TM}}$ and sent to the serial port of a PC as an ASCII message at the same time that the video signal was displayed on a video capture card. Using a system called Real-Time GIS the positions are displayed in the ArcView $^{\mathrm{TM}}$ GIS in precise synchronisation with the video display (McCarthy, Raper, \& Cooper, 1997). This makes it possible to capture time-labelled frames containing phenomena of interest into digital form from the displayed analogue video, which can then be positioned by cross-referencing the time of capture to the position of the camera platform at that time. This makes it possible to create a multimedia spatial database ("a virtual environment") of many digital video frame grabs where the position and time of the capturing platform are stored for each grabbed frame. It is now possible to query the video by time and see the corresponding positions or to query a given location and see the corresponding images.

\section{Positioning, Attitude and Ranging Systems}

Having captured video imagery into digital form and assigned a position to the camera platform for the time when the video image was recorded, the next step is to recover the position of any target phenomena visible in the imagery. This is one of the more complex but key areas and requires recording the camera's position and attitude while ranging the location of the target. This is not a trivial task as the platform can be moving in three dimensions with respect to a target. There is a direct relationship between the precision of the remote position determination and the resulting cost/technical complexity of the solution. The current research aims to produce $10-20 \mathrm{~m}$ positional accuracy for targets visible in oblique imagery from low elevations. This requires positioning recorded using differential GPS which is corrected to produce $2-5 \mathrm{~m}$ accuracy for $1 \mathrm{~s}$ updated positions. The differential correction is provided in real-time using the carrier wave of a commercial FM radio signal broadcast in the U.K.

Attitude information has been recorded using a TCM2 flux-gate magnetometer (Precision Navigation, 1997). This instrument is a high-performance, low-power electronic compass sensor that outputs compass heading, pitch and roll readings via an electronic interface to a host system. It is based upon a proprietary triaxial magnetometer system and a biaxial electrolytic inclinometer and contains no moving parts. Initial calibration for hard iron (e.g., magnets) and soft iron (e.g., ferrous metals) effects needs to be carried out before use. It is usually operated in strapdown mode (i.e., fixed with respect to the camera platform). Accuracy in pitch, roll and yaw is quoted in the range 0.4-1.5 degrees, however, care must be taken when calibrating the instrument so that magnetic anomalies caused by hard iron sources are removed (Titterton \& Weston, 1997). 
Range distance is provided by a laser ranger, in this case, LTI's Impulse. The type of laser used in this instrument is an infrared semiconductor laser diode. The generated light energy has a wavelength of approximately $900 \mathrm{~nm}$, with a beam divergence of $3 \mathrm{mrad}$, equal to a beam width of about $3 \mathrm{~m}$ at 1,000-m distance and target acquisition times range from 0.3 to $0.7 \mathrm{~s}$. This instrument can range targets to a maximum distance of $575 \mathrm{~m}$ : range accuracy for a grey target at $150-\mathrm{m}$ distance is quoted as $5 \mathrm{~cm}$ within one standard deviation (LTI, 1997). In the data acquisition exercise carried out for this application the Hi-8 video camera was clamped to the LTI Impulse laser ranger which was in turn bolted to the TCM2 attitude sensor.

\section{Datalogging}

The data acquisition components described above feed data into the datalogging module as shown in Figure 1. A portable computer was used to record the position, attitude and range data along with the same GPS data that was sent to the Telenav ${ }^{\mathrm{TM}}$ encoder. Software has been developed to handle the synchronisation and logging of the positioning, attitude and ranging information. This has been implemented for a portable computer running Microsoft $^{\mathrm{TM}}$ Windows $^{\mathrm{TM}}$ (200 MHz, 32MB RAM) using a 4-way multi-port serial data PCMCIA card (in this case the QSP-100; Quatech, 1997). The QSP-100 provides four independent asynchronous serial communication interfaces which allow the positioning, attitude and ranging to be recorded simultaneously and recorded at high speed). Typically GPS is output at $1 \mathrm{~Hz}$, attitude information from TCM 2 can be recorded at $40 \mathrm{~Hz}$, with the laser range instrument usually being triggered by the user. A number of issues including latency and datum transformation (Ordnance Survey, 1997) will be described in a future paper.

\section{Aerial Videometry}

Aerial videometry can be defined as the procedures used to extract robust georeferenced measurements of the position of target phenomena visible in aerial video imagery using orientation parameters recorded at the time of acquisition (Figure 2). Aerial videometry

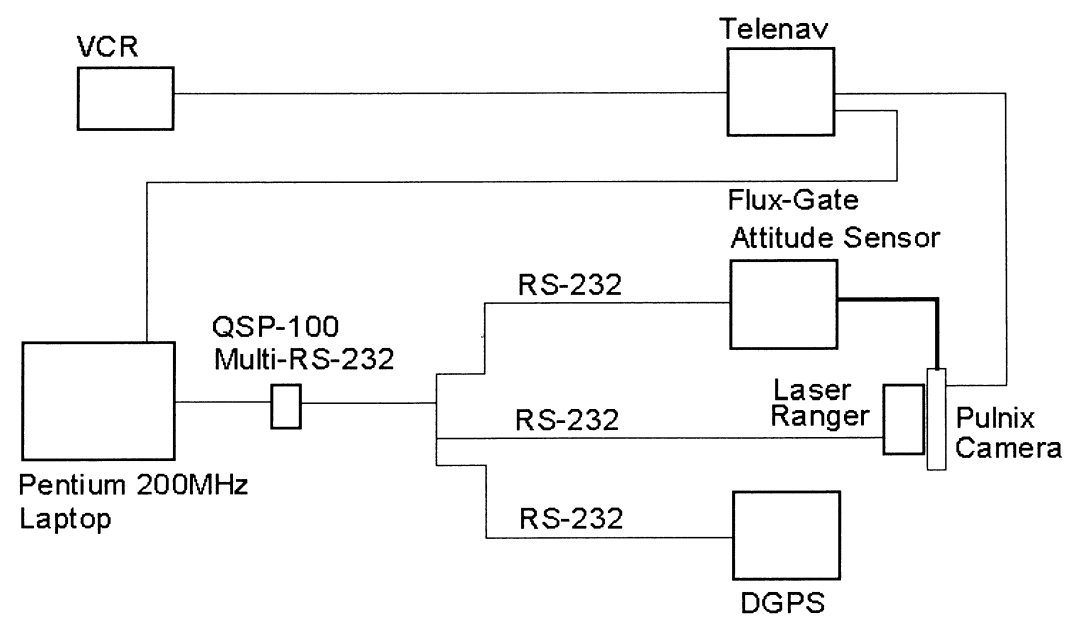

FIGURE 1. Data acquisition system used to collect data from aerial platforms. 


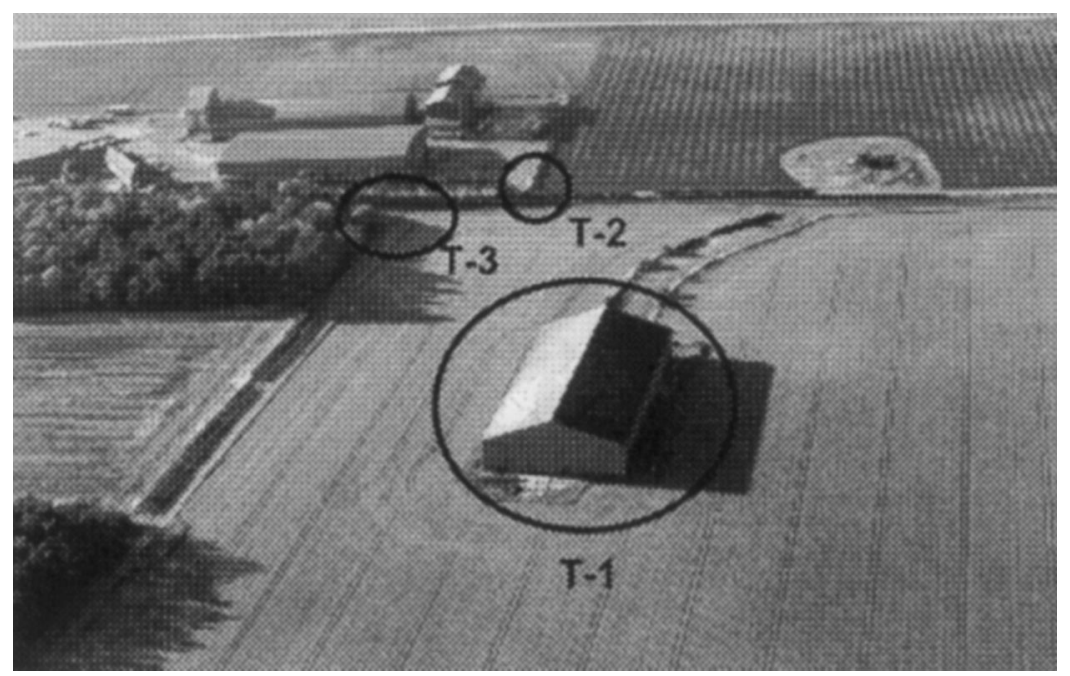

FIGURE 2. Typical targets acquired during aerial videometric testing: T-1: building, T-2: field boundary, T-3: road intersection.

allows the remote measurement of objects (i.e., there is no need establish ground control which may be costly, hazardous or, in some cases, impossible). Taken together, precise positioning, camera platform attitude and target ranging provide the necessary measurements by which aerial videometry can be carried out.

A number of algorithms are employed to extract real-world coordinates from the video frame including simple trigonometry (Sullivan, 1996) and conventional photogrammetry methods (Wolf, 1983). In effect, videometry procedures calculate the horizontal and vertical offsets from the platform position to the target visible in the image. This extends the available positioning for the target phenomena from their nominal position at the camera platform to their true position. This system is still completing calibration and field tests on measurements of single point, line and area objects (Figure 2). Positioning targets using a conventional camera and a more robust navigation instrument such as the Ashtech 3DF system from a fixed wing aircraft can produce positional accuracies of $50 \mathrm{~cm}$ (Corbett \& Short, 1993). In this study, the much lower cost aerial videometry system described has achieved accuracies within the 10-20 m range specified, making a useful order of precision available to a wider range of users.

Aerial videometry offers new potential for the creation of virtual environments based on video imagery providing a high level of data currency; that is, timely data, greater completeness and the ability to record any target phenomena of interest (Novak, 1995). As long as the video camera conforms to Comite Consultatif International des Radiocommunications (CCIR) standards there is no reason why infrared or even thermal cameras (Amin \& Petrie, 1993) cannot be plugged into such a system. Aerial videometry is low cost and also compact as a typical system weighs less then $12 \mathrm{~kg}$ and can fit into a $30-\mathrm{L}$ rucksack. The development of this trial application shows how sophisticated virtual environments can be constructed when made up of multiple generations of video imagery, all stored with full spatial and temporal referencing, a task which no current GIS can fulfil. 


\section{COMPONENT TECHNOLOGIES FOR A GEOREFERENCED VIRTUAL WORLD}

To create a virtual environment which links a user-browseable virtual world with a digital map requires the instantaneous determination of the users' field of view in the virtual world so that it can be shown on a corresponding map. VR systems support the creation of views onto a scene from any position, making it possible to create multiple views of the same virtual world simultaneously, each possibly using different scales. By contrast GIS present a vertically viewed, synoptic view of geographic data, usually oriented to the north or toward a map datum. While the virtual world mimics the human view, the (paper) map is more often used to become familiar with new environments. Presenting both map and virtual world views simultaneously makes the virtual environment easier to comprehend.

\section{Virtual Reality}

While VR has its roots in the 1950s and 1960s (Kalawsky, 1993), concerted research only began in the 1980s when computer processor power became adequate to allow the creation of realistic virtual worlds (Earnshaw et al., 1993). The first experiments with the use of VR to create geographic representations began in the early 1990s (Raper \& McCarthy, 1994b) with work to develop data structure translators from GIS to VR formats. VR systems can now be subdivided into two different groups: firstly, "immersive" (or egocentric) VR which uses a helmet-mounted video/audio system to give the user the experience of interactive immersion within the virtual world; and, secondly, desktop, "through-thewindow" (or exocentric) VR which displays the virtual world on a traditional 2D monitor screen. VR system users control their interaction with the environment using a mouse, "spaceball" or data glove device to issue navigation and selection commands. At the time of writing through-the-window VR systems which can be accessed without special headgear dominate the systems in use, although the high data volumes required to draw and shade the triangular facets making up the virtual world require high-end PCs with high performance graphics display cards.

One of the most widely used forms of through-the-window VR is based upon the use of VR functionality "plug-ins" to Web browsers which can read and display virtual worlds based on the Virtual Reality Markup Language (VRML, 1996). These plug-ins are written as pieces of software which can operate alongside the main browser application and are called upon when required. In the Microsoft ${ }^{\mathrm{TM}}$ Windows $^{\mathrm{TM}}$ operating system these plug-ins usually take the form of ActiveX "controls" (with .ocx file extensions; Microsoft, 1997).

The creation of virtual "geographic" worlds made up of terrains draped with map data or remotely sensed imagery requires a particularly large number of shaded triangular facets to represent the land surface shape, often more than 5,000. Level-of-detail quadtree algorithms have been developed to help solve this basic problem so that only those objects near to the user's current viewing position are displayed using enough triangular facets to give high resolution (Muchaxo, 1998). In virtual geographic world creation, it is also possible to reduce the number of triangles used to form the terrain by using filtering techniques. These techniques reduce the number of triangles making up the terrain by amalgamating them when the angles subtended vertically between adjacent triangles are small. To establish the georeferencing of the virtual world requires that the VR system coordinates which default to $0,0,0$ at the centre of the world are set to real-world 
coordinates. In addition to the static components of the virtual world, dynamic components which move (e.g., aerial vehicles) can also be tracked and displayed.

\section{Geographic Information Systems}

To construct a virtual environment linking a view onto a virtual world with a map requires a GIS which is capable of communicating in real-time with the VR system through an interapplication communication protocol such as one of those provided by Microsoft ${ }^{\mathrm{TM}}$ Windows ${ }^{\mathrm{TM}}$. The GIS must also be able to position and plot the field of view (FOV) onto a virtual world from the user's viewing position. This allows the VR system to send information on the viewing perspective to the GIS which will then automatically update the map. If there are dynamic phenomena which need to be mapped as they move, then the GIS should be able to handle rapid inputs and store positions with associated attributes.

The GIS can also import and map any georeferenced data in geometric or attribute form so the user can see the correspondences between the virtual world and any selected thematic layer. GIS with this level of functionality are now available in the form of ActiveX controls which can interoperate with VR controls. This is known as software component design.

\section{VFC-VRGIS}

The Virtual Field Course (VFC) Project was established in September 1996 as a joint development project between Birkbeck College, Leicester, and Oxford Brookes Universities and funded by the Joint Information Systems Committee of the U.K. Higher Education Funding Councils. The VFC aims to deliver advanced visualisation and mapping tools using georeferenced VR and multimedia in an attempt to augment and extend existing field teaching in disciplines such as geography, geology and ecology. The main objective of this project is the development of virtual fieldwork software tools to support learning, however, the concept may also prove applicable for remote inspection and auditing for utilities and for planning scenario development. It was decided to implement a virtual environment integrating VR and GIS as a visualisation and browsing interface to the database of georeferenced information available for two prototype development areas in N. Norfolk and Devon in England.

The prototype system, VFC-VRGIS (Figure 3) is made up of two main windows; VR (right) and GIS (left). In the application illustrated here there is a dynamic element in the virtual world, viz. an aerial survey plane. The survey plane's position is denoted by the black circle and camera angle by the black line in the VR window. The rectangle in the GIS window displays the aircraft position on 2D map data. Below the GIS window is a box showing which thematic layer is currently displayed in the GIS and indicating the attributes of any map feature which the user queries.

VFC-VRGIS has been implemented using 32-bit Microsoft ${ }^{\mathrm{TM}}$ Visual Basic, which allows the user to incorporate existing ActiveX controls into a single unified application. Industry standard software components have been used so ensuring longevity/stability and also in order to avoid reinventing functionality. This means that the software engineer can use existing software or controls such as ESRI's MapObjects ${ }^{\mathrm{TM}}$ control for GIS functionality and Sense8's control for VR in a shared environment. MapObjects ${ }^{\mathrm{TM}}$ is a set of mapping and GIS components for application developers consisting of a collection of programmable software tools that allow application developers to add mapping and GIS capabilities to applications (ESRI, 1997). Similarly Sense8 have developed ActiveX 


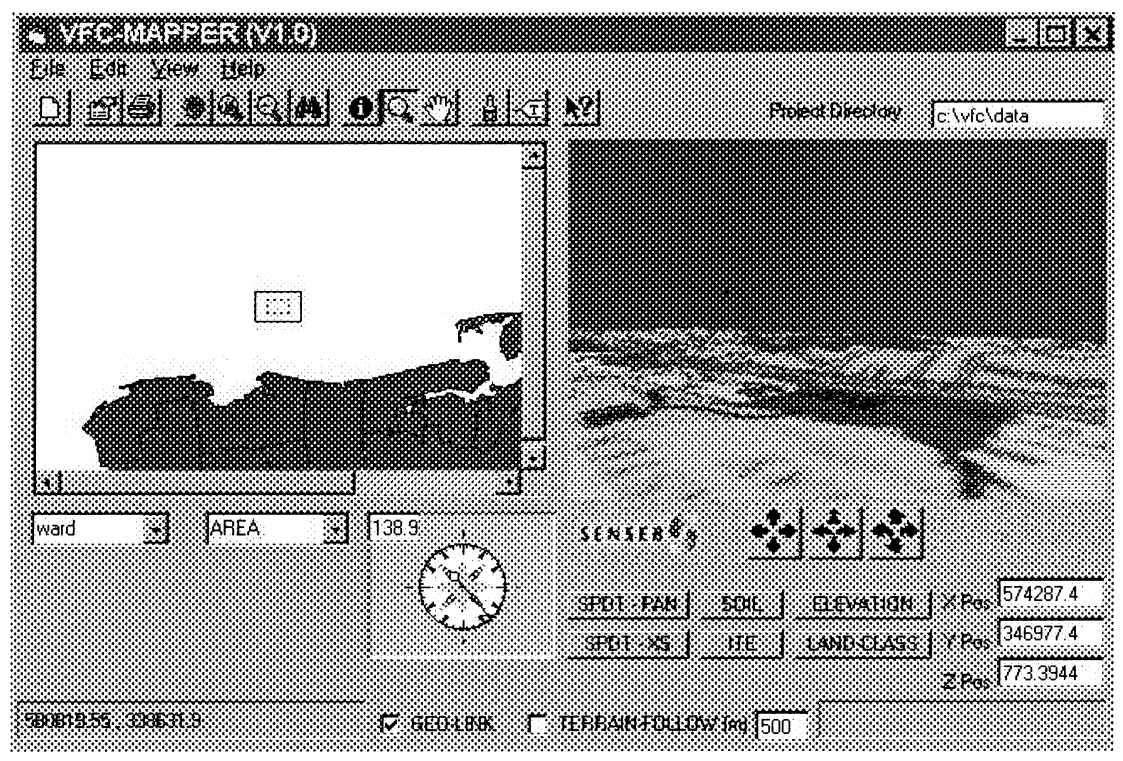

FIGURE 3. The VFC-VRGIS processing system.

controls to provide virtual world functionalities. The two components are geographically interlinked so that either of them can control the other. For example, the VR component can move the map around to reflect the user's FOV dynamically. Another option is to "fly" through the virtual world at a predetermined height above the ground while monitoring position on a linked map.

\section{CONCLUSIONS}

This paper has introduced the concept of the "virtual environment": multidimensional/ multimedia representations of phenomena in natural and built environments permitting the realistic monitoring, analysis and evaluation of the component phenomena. It has not been possible hitherto to create such environments as the static nature of most current GIS technology has precluded such developments. The paper has introduced two applications of virtual environments involving 4D representations and has shown how these new approaches have allowed the user to develop more realistic re-creations of real-world phenomena. In the first case, the linking of video with mapping makes it possible to extract geographic information from a medium with no intrinsic georeferencing using the new techniques of aerial videometry integrated within the virtual environment. In the second application, mapping is linked with a virtual world allowing both views to become interlinked. This will allow users to switch back and forwards between a synoptic map interface and a "flythrough" perspective. Both applications are complete working software tools, although in prototype form.

Delivering such virtual environment applications has proved difficult and timeconsuming at this point as the datasets from which to create the applications have had to be collected at first hand. In this research, the aerial video data was captured using a 
light plane, but the approach could clearly be extended to helicopters or even unmanned aerial vehicles which may increasingly be used to gather spatial data (Shustav, Serokhvostov, \& Ivanov, 1997). The software environment has also had to be designed from scratch and has had to use current technology even though the situation is changing very quickly. For example, in the case of video at present most video capture is analogue, but the move to a completely digital data format lifecycle is only a short time away and this will require some design adjustments to the work described here. The applications have also been developed as "stand-alone" solutions but since the ActiveX controls can easily be implemented in a network environment it is possible to see such applications as being delivered over the Internet in the future.

Furthermore, it is suggested in this paper that the approaches demonstrated here are indicative of what the next generation of geographic information handling might look like. The applications are conceptually and practically "open systems" in design and are capable of integrating with many other forms of geographic information handling. The challenge now is to design new forms of integration between the growing forms of georeferenced multidimensional and multimedia data types.

ACKNOWLEDGEMENTS - The Virtual Field Course project has been funded by the U.K. Joint Information Systems Committee of the University Funding Councils and the authors acknowledge the contribution of the other members of the research team.

\section{REFERENCES}

Amin, A. M., \& Petrie, G. (1993). Geometric calibration of thermal video frame scanners. International Society of Optical Engineering, Videometrics II, 2067, 44-54.

Cooper, R., McCarthy, T., \& Raper, J. F. (1995). Airborne videography and GPS. Earth Observation, 4(11), 53-55.

Corbett, S. J., Short, T. M. (1993). Development of an airborne positioning system. Photogrammetric Record, 15(85), 3-15.

Earnshaw, R. A., Gigante, M. A., \& Jones, H. (1993). Virtual reality systems. London: Academic Press.

ESRI. (1997). MapObjects specification [On-line]. Available: http://www.esri.com/base/products/mapobjects

Kalawsky, R. S. (1993). The science of virtual reality. Reading: Addison-Wesley.

LTI. (1997). Impulse specification [On-line]. Available: http://www.lasertech.com/laserproducts/impulse.html

King, D. J. (1995). Airborne multi-spectral digital camera and video sensors: A critical review of systems designs and applications. Canadian Journal of Remote Sensing, 21(3), 245-273.

Mausel, P. W., Everitt, J. H., Escobar, D. E., \& King, D. (1992). Airborne videography: Current status and future perspectives. Photogrammetric Engineering and Remote Sensing, 58(8), 1189-1195.

McCarthy, T., Raper, J., \& Cooper, R. (1997). Real time positioning of geographical features in airborne videography. In The Third International Conference in Airborne Remote Sensing (Vol. II, pp. 241-247). Environmental Research Institute of Michigan, USA.

Microsoft. (1997). ActiveX controls [On-line]. Available: http://www.microsoft.com/workshop/prog/controls/

Muchaxo, J. (1998). Multi-scale representation for large territories. In A. Camara \& J. Raper (Eds.), Spatial multimedia and virtual reality. London: Taylor \& Francis.

Navtech. (1997). Telenav installation and user guide. Market Harborough, UK: Navtech Systems.

Novak, K. (1995). Mobile mapping technology for GIS data collection. Photogrammetric Engineering and Remote Sensing, 61(5), 493-501.

Ordnance Survey. (1997). National Grid/ETRF89 transformation parameters (Geodetic Information Paper No. 2 11/1997, Version 2.2). Southampton, U.K.: Author.

Paragee, R. (1997). Four ways to link GPS position data to airborne video recordings. In The Third International Conference in Airborne Remote Sensing (Vol. II, pp. 209-216). Environmental Research Institute of Michigan, USA.

Quatech. (1997). QSP-100 Four Channel Asynchronous RS-232-PCMCIA Adaptor [User manual]. Ohio, USA

Precision Navigation. (1997). TCM2 Electronic Compass Module (1997) User's Manual (Revision 1.03). Mountain View, CA, USA. 
Raper, J. F. (1995). Making GIS multidimensional. In Proceedings of the First Joint European Conference on Geographic Information (Vol. 1, pp. 232-240). European GIS Foundation.

Raper, J. F., \& McCarthy, T. (1994a). Using airborne videography to assess coastal evolution and hazards. In Proceedings of the European GIS Conference '94 (pp. 1224-1228). European GIS Foundation.

Raper, J. F., \& McCarthy, T. (1994b). Virtually GIS: The new media arrive. In Proceedings of the Association for Geographic Information Conference '94 (pp. 18.1.1-6). Association for Geographic Information.

Raper, J. F., \& McCarthy, T. M. (1997). Integration of real-time GIS data with Web-based virtual worlds. In Proceedings of Association for Geographical Information Conference '97 (pp. 3.5.1-3.5.3). Association for Geographic Information.

Shustav, A. V., Serokhvostov, S. V., \& Ivanov, S. V. (1997). Several aspects of UAV utilizing for airborne environment monitoring. In The Third International Conference in Airborne Remote Sensing (Vol. II, pp. 699-706). Environmental Reasearh Institute of Michigan, USA.

Sullivan, M. (1996). Trigonometry. Upper Saddle River, NJ: Prentice-Hall.

Titterton, D. H., \& Weston, J. L. (1997). Strapdown inertial navigation technology. London: Peter Peregrinus on behalf of IEE.

VRML. (1996). VRML 2.0 specification [On-line]. Available: http://webspace.sgi.com/moving-worlds

Wolf, P. R. (1983). Elements of photogrammetry. New York: McGraw-Hill. 